\title{
SFR and Abundances of Nearby Galaxies
}

\author{
Marianne Takamiya ${ }^{1}$ Daniel Berke $^{2}$, Forrest Bremer ${ }^{1}$, Casey Jones ${ }^{1}$, \\ and Guillaume Poquet ${ }^{3}$ \\ ${ }^{1}$ Department of Physics and Astronomy, University of Hawai'i Hilo, \\ 200 Kawili Street, Hilo, HI 96720, USA \\ email: takamiya@hawaii.edu \\ ${ }^{2}$ EOA, 660 N. A'ohoku Place, Hilo, HI 96720, USA \\ ${ }^{3}$ ESTACA, Campus-Ouest, Rue Georges Charpak, BP 76121 \\ Box 53061 Laval Cedex 9, France
}

\begin{abstract}
We present star formation rates and nebular abundances of 59 different star-forming regions in 16 nearby galaxies. The star-forming regions were selected to be bright in $\mathrm{H} \alpha$ and were observed with the SNIFS integral field spectrograph on the UH $2.2 \mathrm{~m}$ telescope. The spectra span the wavelength range between $3200 \AA$ and $1 \mu \mathrm{m}$. We find that the local star formation rates depend on the local abundances in that low SFRs show a dependence but high SFR appear insensitive to it.
\end{abstract}

Keywords. galaxies: abundances, galaxies: ISM

\section{Introduction}

Star-forming regions $\mathrm{s}$ in galaxies are traditionally identified in the disks of galaxies wherever $\mathrm{H} \alpha$ narrow-band emission is detected. Following this tradition, we have identified 59 different star-forming regions that have detected $\mathrm{H} \alpha$ emission and present here their star-formation rates and abundances. Using SNIFS, a $6 \times 6 \operatorname{arcsec}^{2}$ integral field unit on the UH $2.2 \mathrm{~m}$ telescope, that spans a wide range in wavelengths, we are able to obtain multiple emission lines to determine the abundances, star formation rates and extinction values at each star-forming region. The determination of the physical properties of the ISM in extragalactic sources is generally obtained from long-slit spectroscopy thus obtaining luminosity-weigthed properties over the entire galaxies. Studies of multislit spectra of individual extragalactic ISM are able to present trends of the physical properties of gas as it varies with galactocentric radius. Our study is different from the former and slightly more powerful than the latter, in that each star-forming region is studied over areas of $6 \times 6 \operatorname{arcsec}^{2}$ larger than what a single slit can cover (0.5-1 arcsec) and the spectrograph we use spans a wide range in wavelength capabale of delivering lines from $3200 \AA$ And $1 \mu \mathrm{m}$ thus allowing for an extremely complete set of line diagnostics for extinction, star formation rates, and abundances.

\section{Data Acquisition}

The spectra were obtained with the UH2.2m telescope on Maunakea between 2009 and 2012 using SNIFS (Aldering et al. 2002). Each SNIFS observation produces 225 individual spectra covering a FOV of $6 \times 6$ sq-arcsec. Each individual lenselet delivers a spectrum of a $0.4 \times 0.4$ sqarcsec area within the SNIFS FOV. When the entire FOV contained emission from the HII regions, an offset to a blank region was observed to subtract the sky background. The exposures of all observations were 20-min with the fainter regions observed 2 or 3 times, some observed over more than one night.

\section{Data Reduction}

SNIFS is a very flexible instrument more so considering that it was built to observe exclusively SNe (i.e. point sources). With help from the SN Factory Team (Aldering and Thomas), our 
data were partially reduced by the SNIFS pipeline but the sky subtraction was done separately. UHH undergraduate student Daniel Berke developed a python pipeline from the ground up that measured the fluxes in $\mathrm{H} \alpha$ and $\mathrm{H} \beta$ in the spectra delivered by the SNIFS data reduction. If $\mathrm{H} \alpha$ was not detected at the level of 1 times the sky noise, the spaxel was considered to be a sky spectrum. In each cube, these sky spectra were averaged and then subtracted to each spaxel in the cube. When a cube did not contain any sky spaxels, we used the offset observation to correct for the sky background. After sky subtraction, the following lines were automatically measured: $[\mathrm{OII}] \lambda 3727,[\mathrm{OI}] \lambda 4363, \mathrm{H} \beta,[\mathrm{OIII}] \lambda 4959,[\mathrm{OIII}] \lambda 5007, \mathrm{H} \alpha,[\mathrm{SII}] \lambda 6717$, $[\mathrm{SII}] \lambda 6732$, [SIII] $\lambda 9069$, and [SIII] $\lambda 9532$. The Balmer Decrement, i.e. the ratio between the flux in $\mathrm{H} \alpha$ and $\mathrm{H} \beta$ : $\mathrm{F}(\mathrm{H} \alpha) / \mathrm{F}(\mathrm{H} \beta)$ and a proxy for extinction, the SFR from the $\mathrm{H} \alpha$ flux, and the nebular abundances were determined for each of the spaxels.

\section{Atmospheric Dispersion}

We encountered a roadblock early in this project when we found that a number of spectra showed Balmer ratios less than 2.86. Roughly $30 \%$ of the spectra fell in that category. After several iterations with the python pipeline and crowd-sourcing the inspection of the spectra with the help of $15 \mathrm{UH}$ Hilo freshman undergraduate students, student Forrest Bremer was key in solving the problem that implicated atmospheric dispersion (AD) for the unphysical Balmer line ratios. We are rebuilding the database by rerunning the pipeline on the corrected datacubes shifted by the magnitude of the refraction along the parallactic angle. AD is an important effect especially for sites with extremely good image quality as is Maunakea.

\section{Preliminary Results}

The SFRs and nebular abundances are minimally affected by the AD as we chose to calculate $12+\log (\mathrm{O} / \mathrm{H})$ from the $[\mathrm{NII}] \lambda 6583$ and $\mathrm{H} \alpha$ line ratios (N2 index) using the calibration of Pettini \& Pagel (2004). The SFR are measured in individual spaxel and are thus small, however, when integrating over the HII regions, we obtain values of $1-2 \mathrm{M}_{\odot} / \mathrm{yr}$. A wide range of SFR values are insensitive to the nebular abundance however, the lower SFR values appear to be dependent on the abundance in the sense that lower abundances have a larger SFR. We are currently studying these two star formation modes and their dependence on abundance in addition to correcting for $\mathrm{AD}$.

\section{Future}

The line diagnostics is being fully redone with the help of UH Hilo undergraduate students to correct for the $\mathrm{AD}$ and using the full extend of the emission lines detected. We can not stress enough the need of AD correctors when carrying out spectrocopic studies especially in the era of sub-arcsec FOV with large aperture telescopes.

\section{References}

Aldering, G. et al. 2002, in Proc. SPIE, ed. J. A. Tyson \& S. Wolff, Vol. 4836, p1

Pettini, M \& Pagel, B. E. J. 2004, MNRAS, 348, 59L 